\title{
ORBITS OF RANK ONE AND PARALLEL MEAN CURVATURE
}

\author{
CARLOS OLMOS
}

To the memory of Franco Tricerri

\begin{abstract}
Let $M^{n}(n \geq 2)$ be a (extrinsic) homogeneous irreducible full submanifold of Euclidean space with $\operatorname{rank}(M)=k \geq 1$ (i.e., it admits $k \geq 1$ locally defined, linearly independent parallel normal vector fields). We prove that $M$ must be contained in a sphere. This result toghether with previous work of the author about homogeneous submanifolds of higher rank imply, in particular, the following theorem: A homogeneous irreducible submanifold of Euclidean space with parallel mean curvature vector is either minimal, or minimal in a sphere, or an orbit of the isotropy representation of a simple symmetric space.
\end{abstract}

\section{INTRODUCTION}

The theory of orthogonal representations of Lie groups is very well developed. Nevertheless, very little is known, except for polar representations, about the geometry of the orbits as submanifolds of Euclidean space. In general this geometry is too complicated and may vary from orbit to orbit. Let us describe an example of this situation: the Lie group $\operatorname{Spin}(3)=S^{3}$ acts transitively, with finite isotropy, on the real projective space $\mathbb{P}^{3}$. Using the Veronese embedding $i: \mathbb{P}^{3} \rightarrow \mathbb{R}^{9}, \operatorname{Spin}(3)$ may be regarded as a Lie subgroup of $S O(9)$. In this way, the Veronese submanifold $i\left(\mathbb{P}^{3}\right)$ is a maximal dimensional orbit of $\operatorname{Spin}(3)$. The geometry of this orbit is well understood (in particular, it is an extrinsic symmetric submanifold, [F]). But, the geometry of other orbits seems to be very involved. It may also happen that an involved group representation of a compact Lie group has very simple orbits (e.g. product of spheres; see [D, p. 126]). So, it seems to be more reasonable (from a geometric point of view) to study homogeneous submanifolds with simple geometric invariants, rather than all the orbits of some representation of a Lie group (because the subgroup of isometries of the ambient space that leaves invariant some orbit may depend on the orbit. This is not indeed the case of polar actions, where this subgroup is given by the associated $s$-representation). With this idea in mind it was defined in [03] the concept of rank of a full submanifold $N$ of Euclidean space. Namely, $\operatorname{rank}(N)=\operatorname{dim}_{N}\left(\nu_{0}(N)\right)$, where $\nu_{0}(N)$ is the maximal parallel flat

Received by the editors April 5, 1994.

1991 Mathematics Subject Classification. Primary 53C40; Secondary 53C42.

Supported by Universidad Nacional de Córdoba and CONICET and partially supported by CONICOR and Fundación Antorchas. 
subbundle of the normal bundle $\nu(N)$ (i.e. the maximal number of linearly independent locally defined normal vector fields to $N$ ).

Theorem A ([O3]). Let $M^{n}$ ( $\left.n \geq 2\right)$ be a compact homogeneous irreducible full submanifold of Euclidean space with $\operatorname{rank}(M) \geq 2$. Then $M$ is an orbit of the isotropy representation of a simple symmetric space.

Corollary ([O3]). Let $M$ be a compact homogeneous irreducible submanifold of Euclidean space with parallel mean curvature vector which is not minimal in a sphere. Then $M$ is an orbit of the isotropy representation of a simple symmetric space.

The proof of the above theorem is related to normal holonomy groups [O1] and to the theorem of Thorbergsson [Th] (see also [O2]). It should be remarked that it might be possible for a compact Lie subgroup of $S O(N)$ to have an irreducible full (nonisoparametric) orbit of $r a n k \geq 2$, but its action on $\mathbb{R}^{N}$ being not polar (this is an open question). The main purpose of this article is to analyze the noncompact case

Theorem. Let $M^{n}$ ( $n \geq 2$ ) be a homogeneous irreducible full submanifold of Euclidean space with $\operatorname{rank}(M) \geq 1$ Then $M$ is contained in a sphere.

For the proof of the above theorem, roughly speaking, we have to pass to a holonomy tube with flat normal holonomy (cf. [HOT]) which is in general nonhomogeneous. Some of the curvature normal of this tube are parallel. (Namely, those corresponding to the horizontal lifting of the eigendistributions associated with nonzero eigenvalues of the shape operator of $M$ restricted to $\nu_{0}(M)$ ), and those corresponding to the vertical distribution). The eigendistributions of the shape operator of the tube which are associated to a nonzero parallel curvature normal can be simultaneously focalized (this is by defining a Coxeter group with a fixed point, as Terng [Te] did for an isoparametric submanifold. But our method of finding the fixed point is simpler and it also applies to the isoparametric case, where it was already known by Alan West [W]). With all this information we are able to prove, if $M$ is irreducible, that all curvature normals of $\mathrm{M}$ related to $\nu_{0}(M)$ must be different from zero (otherwise $M$ would split). This implies that $\mathrm{Hol}_{\xi}(\mathrm{M})$ is contained in a sphere, whose center is given by the fixed point of the Coxeter group. Hence, $M$ is contained in a sphere.

Theorem $\mathrm{A}$ is also true if $M$ is not compact but contained in a sphere. So, glueing it together with the above theorem yields

Theorem. Let $M(n \geq 2)$ be a homogeneous irreducible full submanifold of Euclidean space. Then,

(i) $\operatorname{rank}(M) \geq 1$ if and only if $M$ is contained in a sphere.

(ii) $\operatorname{rank}(M) \geq 2$ if and only if $M$ is an orbit of the isotropy representation of a simple symmetric space of higher rank which is not most singular.

The above theorem, in particular, gives a complete answer, up to minimal immersions, to the problem of finding all homogeneous solutions to the PDE system determined by the parallel mean curvature condition.

Corollary. Let $M$ be a homogeneous irreducible submanifold of the Euclidean space with parallel mean curvature vector. Then $M$ is either minimal, or minimal in a sphere, or it is the orbit of the isotropy representation of a simple symmetric space. 
It would be very interesting to prove similar results for homogeneous (noncompact) submanifolds of the hyperbolic space. Though some of the technics developed here apply also to this case, this study seems to be more involved than in the Euclidean space.

We would like to add that we have included in the Appendix a characterization of the action of the (extrinsic) group of isometries of a homogeneous irreducible submanifold of the Euclidean space.

\section{Preliminaries}

We recall here the notation and basic facts in [O3]. If $M$ is a submanifold of Euclidean space, then $\nu_{0}(M)$ denotes the maximal $\nabla^{\perp}$-parallel and flat subbundle of the normal bundle $\nu(M)$ over $M$. The rank of $M$ is defined by $\operatorname{rank}(M)=\operatorname{dim}_{M}\left(\nu_{0}(M)\right)$; in other words, near any point of $M$, there exists $\operatorname{rank}(M)$ (locally defined) linearly independent parallel normal vector fields, and $\operatorname{rank}(M)$ is maximal with respect to this property. For any $p \in M$,

$$
\nu_{0}(M)_{p}=\left\{\xi \in \nu(M): \Phi_{p}^{*} \cdot \xi=\xi\right\}
$$

where $\Phi_{p}^{*}$ denotes the restricted normal holonomy group of $M$ at $p$.

We next announce Theorem $\mathrm{C}$ of [O3]. Following its proof one sees that it is local in nature, and it does not depend on the compactness. So,

Theorem 1.1. Let $M^{n}=K \cdot v(n \geq 2)$ be a homogeneous irreducible full submanifold of $\mathbb{R}^{N}$, where $v \in \mathbb{R}^{N}$, and $K$ is a Lie subgroup of the full group of isometries of $\mathbb{R}^{N}$. Let $k \in K$ and $p \in M$. Then, there exists $c:[0,1] \rightarrow M$ piecewise differentiable with $c(0)=p, c(1)=k \cdot p$ and such that

$$
\left.d k\right|_{\nu(M)_{p}}=\tau_{c}
$$

where $\tau$ denotes $\nabla^{\perp}$-parallel transport. Moreover, the $\nabla^{\perp}$-parallel transport in $\nu_{0}(M)$ along any curve is achieved by some element of $K$.

Theorem $\mathrm{A}$ in [O3] is also true if the submanifold is not compact, but contained in a sphere (see Remark 1.3).

Theorem 1.2 (see [O3]). Let $M=K \cdot v(n \geq 2)$ be a homogeneous irreducible full submanifold of $\mathbb{R}^{N}$, where $K$ is a Lie subgroup (not necessarily compact) of $S O(N)$, and $v \in \mathbb{R}^{N}$. If $\operatorname{rank}(M) \geq 2$ then $M$ is the orbit of the isotropy representation of a simple symmetric space.

Remark 1.3. In the assumptions of the above theorem. It is not difficult to see that we may assume that $K$ is closed, as a subgroup of the isometries $I(M)$ of $M$. So, at any $p \in M$, the isotropy subgroup $K_{p}$ is compact. It is now easy to see that everything in [O3, sec.6] is also valid for $M$ not necessarily compact, but contained in a sphere.

In the following, unless otherwise stated, $M=K \cdot v$ will be a homogeneous irreducible full submanifold of $\mathbb{R}^{N}$ with $\operatorname{rank}(M) \geq 1$ and dimension $n \geq$ 2. As in [O3, sec.6], we may assume, perhaps by passing to a parallel orbit, that $\nu_{0}(M)$ is globally flat. In this case, there exist distinct $\nabla^{\perp}$-parallel $0=$ $n_{0}, n_{1}, \cdots, n_{g} \in C^{\infty}\left(M, \nu_{0}(M)\right), E_{0}, \cdots, E_{g}$, autoparallel distributions of $M$ (perhaps, $E_{0}=0$ ), such that $T M=E_{0} \oplus \cdots \oplus E_{g}$, and

$$
\mathrm{A}_{\xi} X_{i}=\left\langle n_{i}, \xi\right\rangle \cdot X_{i}
$$


for all $\xi \in C^{\infty}\left(M, \nu_{0}(M)\right), X_{i} \in C^{\infty}\left(M, E_{i}\right)(i=0, \cdots, g)$. Observe that due to the Ricci identity, the distributions $E_{0}, E_{1}, \cdots, E_{g}$ are invariant under all the shape operators of $M$ (see [O3]). (Here, in contrast with [O3, sec. 6], 0 may be an eigenvalue, since $M$ is not necessarily contained in a sphere.) We call $n_{0}, n_{1}, \cdots, n_{g}$ the $\nu_{0}(M)$-curvature normals. We assume, without loss of generality, $g \geq 2$ (see Remark 1.6). Let, for $q \in M, S_{i}(q)$ be the integral manifold of $E_{i}$ through $q(i=0, \cdots, g)$, which is totally geodesic in $M$. For $i=1, \cdots, g$, let $\xi_{i} \in C^{\infty}\left(M, \nu_{0}(M)\right)$ be parallel and such that $\left\langle\xi_{i}, n_{j}\right\rangle=1$ if and only if $i=j$. Let $M_{i}$ be the corresponding focal parallel manifold. One sees, by following the proofs, that Proposition 6.3 in [O3] is valid in our case for $i \geq 1$ (i.e., if $n_{i}$ is not zero).

Proposition 1.4. Under the general assumptions and notation of this section. Let $i \geq 1$. Then

$$
S_{i}(q)=\Phi_{i}^{*} \cdot\left(-\xi_{i}(q)\right)
$$

where $\Phi_{i}^{*}$ denotes the restricted normal holonomy group of $M$ at $q+\xi_{i}(q)$

Corollary 1.5. Let $i \geq 1$, and $q \in M$. Then $S_{i}(q)$ is an orbit of an $s$ representation, and hence, a submanifold with constant principal curvatures.

Remark 1.6. We have that $g \geq 1$, otherwise there would exist a parallel normal section $\eta$ of $M$ such that the shape operator $\mathrm{A}_{\eta}=0$. Then, by [E], $M$ would not be full. If $g=1$, and $\operatorname{dim}\left(E_{0}\right)>0$, then, by [M, Lemma] (see [O3]), $M$ would split. If $g=1$, and $\operatorname{dim}\left(E_{0}\right)=0$, then $M$ admits a parallel nonzero umbilical section, which implies that $M$ is contained in a sphere. So, for our purposes, we may assume that $g \geq 2$.

Definition. Let $j: N \rightarrow \mathbb{R}^{m}$ be an immersed submanifold with globally flat normal bundle. A $C^{\infty}$ distribution $E$ on $N$ is called an eigendistribution if for each $p \in N,(E)_{p}$ is a full eigenspace of the commuting family of shape operators of $N$ at $p$.

Associated to any eigendistribution $E$ on the submanifold $N$, there is $n^{E} \in$ $C^{\infty}(N, \nu(N))$, called the curvature normal associated to $E$, which satisfies: $\tilde{\mathrm{A}}_{\xi} X=\left\langle n^{E}, \xi\right\rangle X, \forall \xi \in C^{\infty}(N, \nu(N)), X \in C^{\infty}(N, E)$, where $\tilde{\mathrm{A}}$ denotes the shape operator of $N$. If we assume that $n^{E}$ is a parallel section, then, as in the isoparametric case, the distribution $E$ is autoparallel (and hence, integrable with totally geodesic leaves). Moreover, if $S$ is a leaf of $E$ then $j(S)$ is an extrinsic sphere of radius vector $\|n\|^{-2} n$.

We recall that a vector in the normal space is called focal if 1 is an eigenvalue of the shape operator of this vector. Though we shall only need a variation of the following lemma (see Lemma 3.4), we state it because it is very simple, interesting, and we have not found it in the literature. (It is indeed the key fact for the construction of the Coxeter group associated with the focal affine hyperplanes.)

Lemma 1.7. Let $j: N \hookrightarrow \mathbb{R}^{N}$ be an embedded submanifold with (globally) flat normal bundle and let $\xi$ be a parallel normal vector field to $N$ such that $\xi(x)$ is not focal for any $x \in N$. Let $N_{\xi}$ be the parallel manifold to $N$, i.e. $N_{\xi}=\{x+\xi(x): x \in N\}$. Let $q \in N$ and let $\bar{q}=q+\xi(q)$. Then the affine normal spaces $q+\nu(N)_{q}$ and $\bar{q}+\nu\left(N_{\xi}\right)_{q}$ coincide, as subsets of $\mathbb{R}^{N}$ (we denote 
this subset by $F$ ). Moreover,

$$
\left\{x \in F: x-q \text { is focal in } \nu(N)_{q}\right\}=\left\{x \in F: x-\bar{q} \text { is focal in } \nu\left(N_{\xi}\right)_{q}\right\}
$$

Proof. The fact that $q+\nu(N)_{q}=\bar{q}+\nu\left(N_{\xi}\right)_{q}$ is easy and well known (see [PT]). One has the following relation between the shape operators of $N$ and $N_{\xi}$ (se: [HOT]): $\mathrm{A}_{x-\bar{q}}^{N_{\xi}}=\mathrm{A}_{x-q}^{N} \cdot\left(I d-\mathrm{A}_{\xi(q)}^{N}\right)^{-1}$, for all $x \in F$. Since $x-\bar{q}=(x-q)-\xi(q)$ we have that

$$
\begin{gathered}
\mathrm{A}_{x-q}^{N_{\xi}}=\left(\mathrm{A}_{x-q}^{N}-\mathrm{A}_{\xi(q)}^{N}\right) \cdot\left(I d-\mathrm{A}_{\xi(q)}^{N}\right)^{-1}=\left(\mathrm{A}_{x-q}^{N}-I d+\left(I d-\mathrm{A}_{\xi(q)}^{N}\right)\right) \cdot\left(I d-\mathrm{A}_{\xi(q)}^{N}\right)^{-1} \\
=\left(\mathrm{A}_{x-q}^{N}-I d\right) \cdot\left(I d-\mathrm{A}_{\xi(q)}^{N}\right)^{-1}+I d
\end{gathered}
$$

or equivalently,

$$
\left(I d-\mathrm{A}_{x-q}^{N_{\xi}}\right)=\left(I d-\mathrm{A}_{x-q}^{N}\right) \cdot\left(I d-\mathrm{A}_{\xi(q)}^{N}\right)^{-1}
$$

Hence, $\left(I d-\mathrm{A}_{x-q}^{N_{\xi}}\right)$ is invertible if and only if $\left(I d-\mathrm{A}_{x-q}^{N}\right)$ is invertible.

\section{THE ANTIPODAL MAP}

We keep the notation and assumptions of section 1. By the homogeneity, the distance from $M$ to its focal set is positive.In other words, there exists $\epsilon>0$ such that 1 is not an eigenvalue of the shape operator $A_{\xi}$, for all $\xi \in \nu(M)$ with $\|\xi\|<\epsilon$. Let $\xi \in \nu(M)_{p}$ with $\|\xi\|<\epsilon$, and such that $\Phi_{p} \cdot \xi$ be a principal orbit, where $\Phi_{p}$ denotes the normal holonomy group of $M$ at $p$. Let $\operatorname{Hol}_{\xi}(M)$ be the subset of $\nu(M)$ that one obtains by parallel transporting $\xi$ along any picewise differentiable curve. Then, $\left.i: \operatorname{Hol}_{\xi}(M)\right) \rightarrow \mathbb{R}^{N}$ is an immersion and it has globally flat normal bundle, where $i(q, \xi)=q+\xi$. Moreover, with the metric induced by $i, \mathrm{Hol}_{\xi}(M)$ is a complete Riemannian manifold.

Remark 2.1. The above two facts were proved in [HOT, Theorem B] under the further assumption of being $M$ simply connected. This assumption was only made in order to have a compact normal holonomy group. But the normal holonomy group $\Phi_{p}$ of $M$ at $p$ is contained always in the normalizer (in $\left.O\left(\nu(M)_{p}\right)\right) \tilde{N}$ of $\Phi_{p}^{*}$. Moreover, the connected component $N_{0}$ of the compact Lie group $N=\left\{\left.g\right|_{\left(\nu_{0}(M)_{p}\right)^{\perp}}: g \in \tilde{N}\right\}$ coincides with the connected componet of $\Phi_{p}$, i.e. $\Phi_{p}^{*}$ (see [O1; O3, Lemma 5.2]). Since we assume that $\nu_{0}(M)$ is globally flat we obtain that $\Phi_{p}$ acts trivially on $\nu_{0}(M)_{p}$. Therefore $N_{0} \subset \Phi_{p} \subset N$, which proves that the normal holonomy group of $\mathbf{M}$ is compact.

Let $E$ be an eigendistribution on $\mathrm{Hol}_{\xi}(M)$ with a parallel associated curvature normal. We will define, as Terng [Te] did in the isoparametric case, the antipodal map associated to $E$. But, at this point we would like to point out that there are some difficulties in defining this map globally, due to the fact that we work in the category of immersions. These difficulties also appear for immersed isoparametric submanifolds; though not explicitly remarked in [PT] (but, in this case they can be skipped by defining a local antipodal map). Let us see some facts that we will need for defining the antipodal map $\phi^{E}$ (only in our situation!). Denote by $k$ the dimension (over $\mathrm{Hol}_{\xi}(\mathrm{M})$ ) of $E$, and let $S$ be an integral manifold of $E$. Then $i$ defines an isometric immersion from the complete Riemannian manifold $S$ into the $k$-sphere $i(S)$, and hence, a covering. If $k \geq 2$, then $S$ must be isometric to the simply connected $k$ sphere $i(S)$. If $k=1$ we will also prove that $S$ is isometric to $i(S)$. Being 
$E$ and eigendistribution, $S$ must be vertical or horizontal with respect to the submersion $\mathrm{Hol}_{\xi}(M) \stackrel{p r}{\longrightarrow} M$, where $p r$ denotes the projection to the base (recall that the fibers of this submersion are invariant under the shape operator; see [HOT]). If $S$ is vertical, then $S$ is isometric to $i(S)$, becase the restriction of $i$ to any fiber of $\operatorname{Hol}_{\xi}(M)$ is an embedding into $\mathbb{R}^{N}$. Let us then assume that $S$ is horizontal, and let $\gamma: \mathbb{R} \rightarrow \operatorname{Hol}_{\xi}(M)$ be a geodesic which parametrizes $S$. Then $\gamma(t)=(p r \circ \gamma(t), \zeta(t))$, where $\zeta(t)$ is a parallel normal vector field to $\operatorname{Hol}_{\xi}(M)$ along $\gamma(t)$ (see [HOT]). We have that $i \circ \gamma$ parametrizes the circle $i(S)$ by a constant multiple of its arc length. Let $\tau$ be the least period of $i \circ \gamma$. Then $\left.\zeta\right|_{[0, \tau]}$ may be regarded as a parallel normal vector field to $i(S)$ along the curve $\left.i \circ \gamma\right|_{[0, \tau]}$. Since the (extrinsic) circle $i(S)$ has globally flat normal bundle we conclude that $\zeta(\tau)=\zeta(0)$. Then, since $p r \circ \gamma(0)+\zeta(0)=i \circ \gamma(0)=i \circ \gamma(\tau)=p r \circ \gamma(\tau)+\zeta(\tau)$, we conclude that $p r \circ \gamma(0)=p r \circ \gamma(\tau)$, and hence $\gamma(0)=\gamma(\tau)$ (recall that $M$ is 1-1 immersed). Then $\gamma$ is periodic with period $\tau$ (because $S$ is a leaf of a distribution). This implies that $i: S \rightarrow i(S)$ is not only a covering, but an isometry. We are finally able to define the antipodal map $\phi^{E}: \operatorname{Hol}_{\xi}(M) \rightarrow \operatorname{Hol}_{\xi}(M)$. Let $q \in \mathrm{Hol}_{\xi}(\boldsymbol{M})$, and let $S$ be the integral manifold of $E$ through $q$. Then $\phi^{E}(q)$ is defined to be the antipodal point in the sphere $S$ of the point $q$. This map is of class $C^{\infty}$. Moreover, it is a diffeomorphism since $\phi^{E} \circ \phi^{E}$ is clearly the identity map.

\section{THE COXETER GROUP}

We keep the notation and assumptions of $\S 1$. In this section we will associate, as Terng [Te] did in the isoparametric case, a Coxeter group to $\mathrm{Hol}_{\xi}(M)$. This Coxeter group will depend on all the (global) eigendistributions whose curvature normals are parallel and nonzero. The construction of the Coxeter group is exactly the same as Terng's, but our method of finding the fixed point (for the affine focal set) is different, quite simple, and applies also to the isoparametric case.

For convenience, we recall and state some notation.

Notation. pr: the projection to the base from $\mathrm{Hol}_{\xi}(M)$ to $M$.

$i$ : the immersion from $\operatorname{Hol}_{\xi}(M)$ into $\mathbb{R}^{N}$ defined by $i\left(\left(s, \xi_{s}\right)=s+\xi_{s}\right.$.

$\tilde{\xi}$ : the parallel normal section of $\operatorname{Hol}_{\xi}(M)$ defined by $\tilde{\xi}(q)=\operatorname{pr}(q)-i(q)$.

A : the shape operator of $M$.

$\tilde{\mathrm{A}}:$ the shape operator of $\operatorname{Hol}_{\xi}(M)$.

$\mathscr{H}$ : the horizontal distribution of $\mathrm{Hol}_{\xi}(M)$.

$\nu$ : the vertical distribution of $\operatorname{Hol}_{\xi}(M)$.

$\hat{E}_{i}:$ the unique distribution on $\operatorname{Hol}_{\xi}(M)$ such that $\hat{E}_{i} \subset \mathscr{H}$ and $d(\operatorname{pr})\left(\hat{E}_{i}\right)=$ $E_{i}, i=0, \cdots, g$.

$\hat{E}:$ the distribution $\hat{E}_{1} \oplus \cdots \oplus \hat{E}_{g}=\hat{E}_{0}^{\perp}$.

$\mathscr{F}$ : the family of all global eigendistributions $E$ on $\mathrm{Hol}_{\xi}(M)$ with a parallel nonzero associated curvature normal $n^{E}$.

$H_{q}^{E}:$ the affine subspace $H_{q}^{E}=q+\left\{x \in \nu\left(\operatorname{Hol}_{\xi}(M)\right)_{q}:\left\langle n^{E}(q), x\right\rangle=1\right\}$ of $\mathbb{R}^{N}(E \in \mathscr{F})$.

Lemma 3.1. Let $E_{i}, i \geq 1$, be an eigendistribution of the shape operators of $M$ restricted to $\nu_{0}(M)$. Let $c:[0,1] \rightarrow M$ be a piecewise differentiable curve, 
and let $\psi(t)$ be a parallel normal vector field to $M$ along $c$. Then the shape operator $\left.\mathrm{A}_{\psi(t)}\right|_{\left(E_{i}\right)_{c(t)}}$ has constant eigenvalues.

Proof. The property of having constant principal eigenvalues is equivalent to the fact that the higher order mean curvature tensors (in the symmetric tensor algebra of the normal bundle) be parallel (see [S]). So, $c$ may be assumed to be either vertical or horizontal with respect to the submersion $M \rightarrow M_{i}$. With the same arguments as in the proof of Theorem A, cases (a) and (b) of [O3], we prove the lemma.

Lemma 3.2. Let $\eta \in C^{\infty}\left(\operatorname{Hol}_{\xi}(M), \nu\left(\mathrm{Hol}_{\xi}(M)\right)\right)$ be parallel. Then $\hat{E}$ and $\nu$ are both invariant under the shape operator $\tilde{\mathrm{A}}$ of $\mathrm{Hol}_{\boldsymbol{\xi}}(M)$. Moreover, $\left.\tilde{\mathrm{A}}_{\eta}\right|_{\hat{E}}$ and $\left.\tilde{\mathrm{A}}_{\eta}\right|_{\nu}$ have both constant eigenvalues.

Proof. For any $x \in \operatorname{Hol}_{\xi}(M), T_{p r(x)} M \subset(d i)_{x}\left(T_{x} H o l_{\xi}(M)\right)$, and $(\hat{E})_{x}=$ $\left(E_{0}^{\perp}\right)_{p r(x)}$. If $q \in \operatorname{Hol}_{\xi}(M)$, there exists a horizontal $\tilde{c}:[0,1] \rightarrow \operatorname{Hol}_{\xi}(M)$ with $\tilde{c}(0)=p, \tilde{c}(1)=q$. Let $c=p r \circ \tilde{c}$, then $\eta(\tilde{c}(t))$ may be regarded as a parallel normal vector field to $M$ along the curve $c(t)$. Let $\xi(t)$ be the parallel transport of $\xi \in \nu(M)_{p}$ along $c$. Then, the shape operator $\tilde{\mathrm{A}}$ is related to the shape operator A of $M$ by the next formula (see [HOT]), which in particular shows the invariance of $\hat{E}$ under the shape operator:

$$
\left.\tilde{\mathrm{A}}_{\eta(\tilde{c}(t))}\right|_{(\hat{E})_{\tilde{c}(t)}}=\left.\mathrm{A}_{\eta(\tilde{c}(t))} \cdot\left(I d-\mathrm{A}_{\xi(t)}\right)^{-1}\right|_{\left(E_{0}^{\perp}\right)_{c(t)}} .
$$

Moreover, $\mathrm{A}_{\eta(\tilde{c}(t))}$ and $\mathrm{A}_{\xi(t)}$ commutes, for all $t \in[0,1]$. In fact, $\eta(\tilde{c}(t))$, and $\xi(t)$ are both perpendicular to the orbit $\Phi_{c(t)} \cdot \xi(t)$ at $\xi(t)$, where $\Phi$ denotes the normal holonomy group of $M$. Then, by the Ambrose-Singer theorem, $\left\langle R^{\perp}(X, Y) \xi(t), \eta(t)\right\rangle=0 \quad \forall X, Y \in T_{c(t)}$, and hence, $\left[\mathrm{A}_{\xi(t)}, \mathrm{A}_{\eta(\tilde{c}(t))}\right]=0$ (by the Ricci identity). Therefore, by Lemma 3.1, the eigenvalues of $\left.\tilde{\mathrm{A}}_{\eta(\tilde{c}(t))}\right|_{(\hat{E})_{\tilde{c}(t)}}$ do not depend on $t$, and, in particular, the eigenvalues of $\left.\tilde{\mathrm{A}}_{\eta(p)}\right|_{(\hat{E})_{p}}$ are exactly the same as those of $\left.\tilde{\mathrm{A}}_{\eta(q)}\right|_{(\hat{E})_{q}}$

With respect to $\nu$, notice that the fibers of $\mathrm{Hol}_{\xi}(M) \stackrel{p r}{\longrightarrow} M$ are isoparametric submanifolds of the normal space of $M$ (see [HOT, p. 168]). The invariance of $\nu$ under the shape operator follows easily from the fact that $\operatorname{ker}\left(I d-\tilde{\mathrm{A}}_{\tilde{\xi}}\right)=\nu$. The proof of the constancy of the eigenvalues of $\left.\tilde{\mathrm{A}}_{\eta}\right|_{\nu}$ is similar to that of Theorem A, cases (a) and (b) of [O3].

Lemma 3.3. The distributions $\hat{E}$, and $\nu$ are both a direct sum of elements of $\mathscr{F}$, for $\xi$ small.

Proof. From Lemma 3.2 we obtain that $\nu=\bigoplus_{h=1}^{m} B_{h}$, and $\hat{E}=\bigoplus_{l=1}^{r} B_{l}^{\prime}$, where each element (which we assume nontrivial) in any of both direct sums fails, eventually, from being an eigendistribution, with a parallel associated curvature normal, only by the fact that it could be properly contained in a full eigenspace of the shape operator $\tilde{\mathrm{A}}$ at $q$, for some $q \in \mathrm{Hol}_{\xi}(M)$. Let $h \in\{1, \cdots, m\}$ (resp. $l \in\{1, \cdots, r\}$ ), let $q \in H_{o} l_{\xi}(M)$ and let $\mathbb{V}_{q}$ be the eigenspace of the shape operator at $q$ which contains $\left(B_{h}\right)_{q}\left(\operatorname{resp} .\left(B_{l}^{\prime}\right)_{q}\right)$. Consider the shape operator $\tilde{\mathrm{A}}_{\tilde{\xi}}$, and observe that $\operatorname{ker}\left(I d-\tilde{\mathrm{A}}_{\tilde{\xi}}\right)=\nu$. Then, $1=\left\langle\tilde{\xi}, \bar{n}_{h}\right\rangle \neq\left\langle\tilde{\xi}, \bar{n}_{l}^{\prime}\right\rangle$, where $\bar{n}_{h}$ (resp. $\left.\bar{n}_{l}^{\prime}\right)$ denotes the curvature normal associated with $B_{h}$ (resp. $\left.B_{l}^{\prime}\right)$. This shows that $\mathbb{V}_{q} \cap\left(B_{l}^{\prime}\right)_{q}=0\left(\operatorname{resp} . \mathbb{V}_{q} \cap\left(B_{h}\right)_{q}=0\right)$ 
for any $1, h$. For proving that $\mathbb{V}_{q}=B_{h}$ (resp. $\mathbb{V}_{q}=B_{l}^{\prime}$ ) it remains only to show, since $T\left(\operatorname{Hol}_{\xi}(M)\right)=\hat{E}_{0} \oplus \hat{E} \oplus \nu$, that $\mathbb{V}_{q} \cap\left(\hat{E}_{0}\right)_{q}=\{0\}$. For it, we have to analyze separately $\nu$ and $\hat{E}$ in cases (a) and (b).

Case (a): $\left(B_{h}\right)_{q} \subset \mathbb{V}_{q}$. Let $\eta \in \nu\left(\Phi_{p r(q)} \cdot(-\tilde{\xi}(q))\right)_{-\tilde{\xi}(q)}$ be with $|\eta|=1$, and such that all the eigenvalues of the shape operator $A_{\eta}^{\prime}$ of the orbit of the normal holonomy group $\Phi_{p r(q)} \cdot(-\tilde{\xi}(q))$ are nonzero. Such an $\eta$ does exist because this orbit is a compact isoparametric submanifold of $\nu(M)_{p r(q)}$. The shape operator $\tilde{\mathrm{A}}_{\eta}$ of $\operatorname{Hol}_{\xi}(M)$ is related to the shape operators $\mathrm{A}_{\eta}$ and $\mathrm{A}_{\eta}^{\prime}$ of $M$ and $\Phi_{p r(q)} \cdot(-\tilde{\xi}(q))$ respectively, by the formulas

$$
\begin{gathered}
\left.\tilde{\mathrm{A}}_{\eta}\right|_{(\nu)_{q}}=\mathrm{A}_{\eta}^{\prime}, \\
\left.\tilde{\mathrm{A}}_{\eta}\right|_{\left(\tilde{E}_{0}\right)_{q}}=\left.\mathrm{A}_{\eta} \cdot\left(I d-\mathrm{A}_{-\tilde{\xi}(q)}\right)^{-1}\right|_{\left(E_{0}\right)_{q}} .
\end{gathered}
$$

Each eigenvalue of $A_{\eta}^{\prime}$ is proportional to $|-\tilde{\xi}|^{-2}=|\xi|^{-2}$. So, each eigenvalue of $\mathrm{A}_{\eta}^{\prime}$ tends to infinity if $|\xi|$ tends to zero. (Observe that for any other fiber $\Phi_{p r(x)} \cdot(-\tilde{\xi}(x))$ we can find, since any two fibers are isometric, $\eta_{x}$ $\in \nu\left(\Phi_{p r(x)} \cdot(-\tilde{\xi}(x))\right)_{-\tilde{\xi}(x)}$ of unit lenght and such that the shape operator of this fiber applied to $\eta_{x}$ has the same eigenvalues as $\mathrm{A}_{\eta}^{\prime}$.) But, on the other hand, as $|\xi|$ tends to zero, the eigenvalues of $\mathrm{A}_{\eta} \cdot\left(I d-\mathrm{A}_{-\tilde{\xi}(q)}\right)^{-1}$ remain bounded (independently of the base point, by the homogeneity of $M$ ). It is now clear, if $\xi$ is small, that $\mathbb{V}_{q} \cap\left(\tilde{E}_{0}\right)_{q}=\{0\}$, for all $q \in \operatorname{Hol}_{\xi}(M)$.

Case (b): $\left(B_{l}^{\prime}\right)_{q} \subset \mathbb{V}_{q}$. Assume that $\mathbb{V}_{q} \cap\left(\hat{E}_{0}\right)_{q} \neq\{0\}$. We shall derive a contradiction. Let $X \in \mathbb{V}_{q} \cap\left(\hat{E}_{0}\right)_{q}$ with $|X|=1$. We may assume, without loss of generality, that there exists $Y \in\left(B_{l}^{\prime}\right)_{q} \cap\left(\hat{E}_{i}\right)_{q}$ with $|Y|=1$, for some $i \geq 1$ (because the distributions $\hat{E}_{i}$ are invariant under the shape operator). Let $\delta$ be such that all of the eigenvalues of the shape operator $\mathrm{A}_{\psi}$ of $M$ are less than 1 , for any $\psi \in \nu(M)$ with $|\psi|<\delta$ (such a $\delta$ exists by the homogeneity of $M)$. Assume $|\xi|<\delta$. One one hand,

$$
\begin{aligned}
\left\langle\tilde{\mathrm{A}}_{n_{i}(p r(q))} Y, Y\right\rangle & =\left\langle\mathrm{A}_{n_{i}(p r(q))} \cdot\left(I d-\mathrm{A}_{-\tilde{\xi}(q)}\right)^{-1} Y, Y\right\rangle \\
& =\left\langle\left(I d-\mathrm{A}_{-\tilde{\xi}(q)}\right)^{-1} Y, \mathrm{~A}_{n_{i}(p r(q))} Y\right\rangle \\
& =\left\langle n_{i}(\operatorname{pr}(q)), n_{i}(\operatorname{pr}(q))\right\rangle \cdot\left\langle\left(I d-\mathrm{A}_{-\tilde{\xi}(q)}\right)^{-1} Y, Y\right\rangle \neq 0
\end{aligned}
$$

because $\left(I d-\mathrm{A}_{-\tilde{\xi}(q)}\right)^{-1}$ is positive definite, since $|-\tilde{\xi}(q)|=|\xi|<\delta$. On the other hand,

$$
\begin{gathered}
\left\langle\tilde{\mathrm{A}}_{n_{i}(p r(q))} X, X\right\rangle=\left\langle\mathrm{A}_{n_{i}(p r(q))} \cdot\left(I d-\mathrm{A}_{-\tilde{\xi}(q)}\right)^{-1} X, X\right\rangle \\
=\left\langle\left(I d-\mathrm{A}_{\tilde{\xi}(q)}\right)^{-1} X, \mathrm{~A}_{n_{i}(p r(q))} X\right\rangle=0 .
\end{gathered}
$$

Hence, $X$ and $Y$ cannot both belong to the same eigenspace.

It remains only to show, for the sake of finishing the proof, that the curvature normal $\bar{n}_{h}$ (resp. $\bar{n}_{l}^{\prime}$ ) is nonzero. We have that $\left.\bar{n}_{h}\right|_{(p r)^{-1}(p r(q))}$ is a curvature normal of the compact isoparametric submanifold $(p r)^{-1}(\operatorname{pr}(q))$ of the normal space $\nu(M)_{p r(q)}$. This proves that $\bar{n}_{h}$ is nonzero (see [PT]). Let now $\eta_{p r(q)} \in$ 
$\nu_{0}(M)_{p r(q)}$ be such that $\operatorname{ker}\left(\mathrm{A}_{\eta_{p r(q)}}\right)=\left(E_{0}\right)_{\operatorname{pr}(q)}$. Then,

$$
\begin{aligned}
\left(\hat{E}_{0}\right)_{q} & =\left(E_{0}\right)_{p r(q)}=\operatorname{ker}\left(\mathrm{A}_{\eta_{p r(q)}}\right)=\operatorname{ker}\left(\tilde{\mathrm{A}}_{\eta_{p r(q)}} \cdot\left(\left.\left(I d-\tilde{\mathrm{A}}_{\tilde{\xi}_{(q)}}\right)\right|_{(\mathscr{H})_{q}}\right)^{-1}\right) \\
& =\operatorname{ker}\left(\left.\tilde{\mathrm{A}}_{\eta_{p r(q)}}\right|_{(\mathscr{H})_{q}}\right)
\end{aligned}
$$

(as subspaces of $\mathbb{R}^{N}$ ). This shows that $\left.\tilde{\mathrm{A}}_{\eta_{p r(q)}}\right|_{(\hat{E})_{q}}$ is invertible, and therefore $\bar{n}_{l}^{\prime}$ is nonzero.

Lemma 3.4. Let $q \in \operatorname{Hol}_{\xi}(M)$ and let $E^{\prime} \in \mathscr{F}$. Then, for any $E \in \mathscr{F}, \phi_{*}^{E^{\prime}}(E)$ belongs to $\mathscr{F}$, and

$$
\bigcup_{E \in \mathscr{F}} H_{q}^{E}=\bigcup_{E \in \mathscr{F}} H_{\phi^{E^{\prime}}(q)}^{E}
$$

where $\phi^{E^{\prime}}$ is the antipodal map with respect to $E^{\prime}$.

Proof. We have, for any $q \in \operatorname{Hol}_{\xi}(M), i\left(\phi^{E^{\prime}}(q)\right)=q+\psi(q)$, where $\psi=$ $2\left|n^{E^{\prime}}\right|^{-2} n^{E^{\prime}}$. Then, if $E \in \mathscr{F}, \operatorname{di}\left(\left(\phi_{*}^{E^{\prime}}(E)\right)_{\phi^{E^{\prime}}(q)}\right)=\operatorname{di}\left(d \phi^{E^{\prime}}\left((E)_{q}\right)\right)=$ $\left(I d-\tilde{A}_{\psi(q)}\right)\left((E)_{q}\right)=(E)_{q}$. Since the shape operators at $\phi^{E^{\prime}}(q)$ and $q$ are related by

$$
\tilde{A}_{\eta}^{\phi^{E^{\prime}}(q)}=\tilde{A}_{\eta}^{q} \cdot\left(I d-\tilde{A}_{\psi(q)}^{q}\right)^{-1}
$$

one obtains that $\left(\phi_{*}^{E^{\prime}}(E)\right)_{\phi^{E^{\prime}}(q)}=(E)_{q}$ is a full eigenspace of the (commuting) family of shape operators at $\phi^{E^{\prime}}(q)$. Moreover, its associated eigenvalue function is $\lambda$, where

$$
\lambda(\eta)=\left(1-\left\langle n^{E}, \psi\right\rangle\right)^{-1}\left\langle n^{E}(q), \eta\right\rangle .
$$

This proves that $\phi_{*}^{E^{\prime}}(E) \in \mathscr{F}$, and that $\left(1-\left\langle n^{E}, \psi\right\rangle\right)^{-1} n^{E} \circ \phi^{E^{\prime}}$ is its associated (parallel) curvature normal. Denote $\phi_{*}^{E^{\prime}}(E)$ by $\bar{E}$, and let $x \in H_{q}^{E}$, i.e., $\left\langle x-q, n^{E}(q)\right\rangle=1$. Then $\left\langle x-\phi^{E^{\prime}}(q), n^{E}\left(\phi^{E^{\prime}}(q)\right)\right\rangle=\langle(x-q)-\psi(q)$, $\left.\left(1-\left\langle n^{E}(q), \psi(q)\right\rangle\right)^{-1} n^{E}(q)\right\rangle=\left(1-\left\langle n^{E}(q), \psi(q)\right\rangle\right)^{-1}\left(1-\left\langle\psi(q), n^{E}(q)\right\rangle\right)=1$. Hence,

$$
\bigcup_{E \in \mathscr{F}} H_{q}^{E} \supset \bigcup_{E \in \mathscr{F}} H_{\phi^{E^{\prime}}(q)}^{E}
$$

Since $q=\phi^{E^{\prime}}\left(\phi^{E^{\prime}}(q)\right)$, the other inclusion is also true.

Proposition 3.5. Under the notation and general assumptions of this section. Let $\mathscr{F}$ be the family of all (global) eigendistributions $E$ of $\mathrm{Hol}_{\xi}(M)$, whose associated curvature normal $n^{E}$ is parallel and nonzero. Then, for any $q \in \operatorname{Hol}_{\xi}(M)$, the refletions of $q+\nu\left(H_{o} l_{\xi}(M)\right)_{q}$ across the hyperplanes $H_{q}^{E}, E \in \mathscr{F}$, generates a finite Coxeter group $W_{q}$, and any two of such groups are (orthogonally) equivalent under the parallel displacement.

Proof. Let $q \in \operatorname{Hol}_{\xi}(M), E^{\prime} \in \mathscr{F}$ and let $\tau$ be the $\nabla^{\perp}$-parallel transport, from $\nu\left(\operatorname{Hol}_{\xi}(M)\right)_{q}$ into $\nu\left(\operatorname{Hol}_{\xi}(M)\right)_{q}$, where $\bar{q}=\phi^{E^{\prime}}(q)$. Let $\bar{\tau}$ be the isometry of the affine subspace $F=q+\nu\left(H_{o} l_{\xi}(M)\right)_{q}=\bar{q}+\nu\left(H o l_{\xi}(M)\right)_{q}$, defined by: $\bar{\tau}(q)=\bar{q}$ and $\left.d(\bar{\tau})\right|_{q}=\tau$. The parallel transport $\tau$, which is independent of the curve joinning $q$ with $\bar{q}$, may be achieved by the parallel transport of a curve, let us denote it by $c$, in the integral manifold $S_{q}$ of $E^{\prime}$ through $q$. Since $i\left(S_{q}\right)$ is a totally geodesic (extrinsic) sphere, which is invariant under the shape operator of $\mathrm{Hol}_{\xi}(M)$, we get that the $\nabla^{\perp}$-parallel transport along $c$, as 
a curve of $\mathrm{Hol}_{\xi}(M)$, coincide with the $\nabla^{\perp}$-parallel transport along $c$, in the normal space to the sphere $i: S_{q} \rightarrow i\left(S_{q}\right)$. (the same argument as in [Te] for the isoparametric case). Then, exactly as in the isoparametric case, one has that $\tau$ is the reflection across the hyperplane, in $\nu\left(H_{o} l_{\xi}(M)_{q}\right.$, perpendicular to $n^{E^{\prime}}(q)$ (identifying $\nu\left(\mathrm{Hol}_{\xi}(M)\right)_{q}$ with $\nu\left(\mathrm{Hol}_{\xi}(M)\right)_{q}$, as linear subspaces of $\left.\mathbb{R}^{N}\right)$. An easy calculation shows that $\bar{\tau}: F \rightarrow F$ is the reflection across the hyperplane $H^{E^{\prime}}(q)$. It is clear, from the definition of $\mathscr{F}$ that the (affine) parallel transport $\bar{\tau}$ sends $\bigcup_{E \in \mathscr{F}} H_{q}^{E}$ into $\bigcup_{E \in \mathscr{F}} H_{\bar{q}}^{E}$. But, on the other hand, by Lemma 3.4, $\bigcup_{E \in \mathscr{F}} H_{q}^{E}=\bigcup_{E \in \mathscr{F}} H_{\bar{q}}^{E}$. Hence, the reflection in $F$ across the hyperplane $H_{q}^{E^{\prime}}$ leaves $\bigcup_{E \in \mathscr{F}} H_{q}^{E}$ invariant, for any $E^{\prime} \in \mathscr{F}$. Then, the reflections across the hyperplanes $\left\{H_{q}^{E}\right\}_{E \in \mathscr{F}}$ generates a finite Coxeter group $W_{q}$. This group, being a finite group of affine transformations, has always a fixed point (here, our argument simplify that of [Te]). It is clear that changing the point $q$ by any other, the Coxeter group conjugates by the parallel transport.

Corollary 3.6. Under the same assumptions and notation as in Proposition 3.5. Then there exists a parallel $\psi \in C^{\infty}\left(\mathrm{Hol}_{\xi}(M), \nu\left(\mathrm{Hol}_{\xi}(M)\right)\right.$ such that $\left\langle\psi, n^{E}\right\rangle$ $=1$, for all $E \in \mathscr{F}$.

Proof. Let $q \in \mathrm{Hol}_{\xi}(M)$ and let $\psi_{q}$ be a fixed point of $W_{q}$. Then $\psi_{q}$ belongs to $\bigcup_{E \in \mathscr{F}} H_{q}^{E}$. If $\psi$ is the parallel normal section with $\psi(q)=\psi_{q}$, then $\left\langle\psi, n^{E}\right\rangle=1$, for all $E \in \mathscr{F}$.

\section{THE PROOF OF THE MAIN RESULT}

We keep the assumptions and notations of previous sections.

Lemma 4.1. Assume that the distribution $\hat{E} \oplus \nu$ on $\mathrm{Hol}_{\xi}(M)$ is integrable. Then,

(i) The distribution $E_{0}^{\perp}=E_{1} \oplus \cdots \oplus E_{g}$ on $M$ is autoparallel.

(ii) $E_{0}=0$.

Proof. We shall show first that $E_{0}^{\perp}$ is integrable. Let $X, Y \in C^{\infty}\left(M, E_{0}^{\perp}\right)$ and let $\tilde{X}, \tilde{Y} \in C^{\infty}\left(\operatorname{Hol}_{\xi}(M), \hat{E}\right)$ be the horizontal lift of $X$ and $Y$ respectively. In particular $\tilde{X}$ and $\tilde{Y}$ are $p r$-related to $X$ and $Y$. Then, $[X, Y]_{p r(q)}=$ $d(p r)\left([\tilde{X}, \tilde{Y}]_{q}\right) \in d(p r)\left((\hat{E} \oplus \nu)_{q}\right)=\left(E_{0}^{\perp}\right)_{p r(q)}$, which proves the integrability of $E$. We have the (orthogonal) splitting $E_{0}^{\perp}=E_{1} \oplus \cdots \oplus E_{q}$, being $E_{1}, \cdots, E_{g}$ autoparallel distributions which are invariant under the shape operator. Then, by the Codazzi identity, $E_{0}^{\perp}$ must be autoparallel. In fact, we must only show that $\nabla_{Y} X \in C^{\infty}\left(M, E_{0}^{\perp}\right)$ for $X \in C^{\infty}\left(M, E_{i}\right), Y \in C^{\infty}\left(M, E_{j}\right)$ and $i \neq j$. Let $\eta \in C^{\infty}\left(M, \nu_{0}(M)\right)$ be parallel and such that $\left\langle\eta, n_{i}\right\rangle \neq\left\langle\eta, n_{j}\right\rangle$. We have $\left(\nabla_{X} A_{\eta}\right) Y=\left\langle\eta, n_{j}\right\rangle \nabla_{X} Y-\mathrm{A}_{\eta}\left(\nabla_{X} Y\right)=\left\langle\eta, n_{i}\right\rangle \nabla_{Y} X-\mathrm{A}_{\eta}\left(\nabla_{Y} X\right)=\left(\nabla_{Y} \mathrm{~A}_{\eta}\right) X$ (using the Codazzi identity). Since $\nabla_{X} Y=\nabla_{Y} X+[X, Y]$, we obtain that $\left(\left\langle\eta, n_{j}\right\rangle-\left\langle\eta, n_{i}\right\rangle\right) \nabla_{Y} X=\mathrm{A}_{\eta}([X, Y])-\left\langle\eta, n_{j}\right\rangle[X, Y]$. This shows, since $E_{0}^{\perp}$ is integrable, and invariant under the shape operator, that $\nabla_{Y} X \in C^{\infty}\left(M, E_{0}^{\perp}\right)$. This proves (i). We have that $E_{0}$ and $E_{0}^{\perp}$ are both autoparallel. But two (orthogonally) complementary autoparallel distributions must be parallel. Hence, $E_{0}$ and $E_{0}^{\perp}$ are both parallel. Since they are also invariant under the shape operator, it is not hard to prove, from [M, Lemma], that $E_{0}=0$ or $E_{0}^{\perp}=0$ (otherwise, the irreducible submanifold $M$ would split; see [O3, Lemma 1.1]). If $E_{0}^{\perp}$ would be trivial then $A_{\psi}=0$ for any parallel $\psi \in C^{\infty}\left(M, \nu_{0}(M)\right)$, and consequently, by [E], $M$ would not be full. 
Proof of the Theorem. Let $\psi$ be as in Corollary 3.7 and let $q \in \mathrm{Hol}_{\xi}(\mathrm{M})$. Then, $(\hat{E} \oplus \nu)_{q} \subset\left(\operatorname{ker}\left(I d-\tilde{\mathrm{A}}_{\psi}\right)\right)_{q}$. On the other hand, $\left.\tilde{\mathrm{A}}_{\psi-\tilde{\xi}}\right|_{\nu}=0$ (see Notation). So, $\psi-\tilde{\xi}$ projects down to a parallel (local) normal vector field $\eta$ to $M$, because it must be constant along the vertical leaves (a similar argument was used in $[\mathrm{O} 2$, p. 229]). Then,

$$
0=\left.\mathrm{A}_{\eta_{p r(q)}}\right|_{\left(E_{0}\right)_{p r(q)}}=\tilde{\mathrm{A}}_{(\psi-\tilde{\xi})(q)} \cdot\left(\left.\left(I d-\tilde{\mathrm{A}}_{\tilde{\xi}(q)}\right)\right|_{\left(\hat{E}_{0}\right)_{q}}\right)^{-1}
$$

and therefore

$$
\left.\tilde{\mathrm{A}}_{(\psi-\tilde{\xi})(q)}\right|_{\left(\hat{E}_{0}\right)_{q}}=0 .
$$

Since any eigenvalue of $\left.\tilde{\mathrm{A}}_{\tilde{\xi}(q)}\right|_{(\mathscr{L})_{q}}$ is different from 1, we conclude, from the above equality, that any eigenvalue of $\left.\tilde{\mathrm{A}}_{\psi}\right|_{\left(\hat{E}_{0}\right)_{q}}$ is different from 1. Hence, $(\hat{E} \oplus \nu)=\operatorname{ker}\left(I d-\tilde{A}_{\psi}\right)$, which is, by the Codazzi identity, an autoparallel distribution and therefore integrable. Then, by Lemma 4.1, we obtain that the distribution $E_{0}$ on $M$ is the null distribution and consequently $\hat{E}_{0}$ is also null. Then, $\psi$ is an umbilical parallel normal section of $\operatorname{Hol}_{\xi}(M)$, which implies that $\operatorname{Hol}_{\xi}(M)$ is contained in a sphere. Since $\left(\operatorname{Hol}_{\xi}(M)\right)_{\xi}=M$, it follows that $M$ is contained in a sphere.

Remark. A nontrivial example of a homogeneous embedded noncompact submanifold of the Euclidean space can be produced as follows: consider the following submanifold $M$ of $\mathbb{R}^{5}=\mathbb{C} \times \mathbb{C} \times R$ defined by

$$
M=\left\{\left(e^{i s}, e^{i t}, 2 s-t\right)\right\} .
$$

Observe that $M$ is the orbit through $(1,1,0)$ of the abelian two dimensional subgroup of isometries of $\mathbb{R}^{6}$ generated by $\phi_{t}$ and $\psi_{t}$, where $\phi_{t}(a, b, c)=$ $\left(e^{i t} . a, b, c-t\right), \psi_{s}(a, b, c)=\left(a, e^{i s} \cdot b, c+2 s\right)$. It is not difficult to check that $M$ is an irreducible full embedded submanifold.

Less trivial examples, i.e. nonintrinsically flat, can be obtained as follows: Let us consider the isotropy representation of a Hermitian symmetric space of complex dimension $n$. Assume that after deleting the $S^{1}$ factor of the isotropy group, this new group does not act any more polarly on $\mathbb{C}^{n}$ (there exist such examples!), and hence it does not act transitively on any principal orbit of the isotropy representation. Let $K \times \mathbb{R}$ be the universal cover of the isotropy group of the hermitian symmetric space, where $K$ is compact. Consider now the following representation $\rho$ of $K \times \mathbb{R}$ on $I\left(\mathbb{C}^{n} \times \mathbb{R}\right)$ defined by $\rho(k, v)=$ $\left(\tilde{\rho}(k, t), \tau_{v}\right)$, where $\tilde{\rho}$ is the obvious representation of $K \times \mathbb{R}$ on the isometry group of $C^{n}$ (i.e., the projection to the isotropy group followed by the isotropy action) and $\tau_{v}$ denotes the translation along $v$. Then, the principal orbits of $K \times \mathbb{R}$ are irreducible full homogeneous noncompact submanifolds. (We have required $K$ not to act transitively on principal orbits because otherwise the principal orbits of $(K \times \mathbb{R}, \rho)$ would split a line.)

\section{APPENDIX}

In this appendix we include a characterization of noncompact homogeneous submanifolds of Euclidean space in terms of the action of its group of (extrinsic) isometries. This characterization, which we have not found in the mathematical literature, generalizes the following result of J. Vargas [V]: "A symmetric space 
of the noncompact type does not admit an equivariant isometric immersion into the Euclidean space".

Let $M=G \cdot v$ be a noncompact irreducible submanifold of $\mathbb{R}^{N}$ ( $G$ a connected Lie subgroup of $\left.I\left(\mathbb{R}^{N}\right)\right)$. Then, the universal cover $\tilde{G}$ of $G$ splits as $K \times \mathbb{R}^{k}$, where $K$ is a (simply connected) compact Lie group. In fact, if $H$ denotes the subgroup of $G$ which consists of all translations, then it is a normal subgroup. The tangent space to the orbits of $H$ in $M$ define a parallel distribution $\mathscr{D}$ on $M$ (because it is the restriction of a parallel distribution on $\mathbb{R}^{N}$ ). Moreover, it is easy to see that it is invariant under the shape operator of $M$, and therefore, by [M, Lemma], $M$ splits, unless $\mathscr{D}=0$ (or $M$ is a straight line). Then, the obvious projection from $G$ into $S O(N)$ is a Lie group morphism whose kernel is discrete, and consequently an immersion. Then, the Lie algebra of $G$ is isomorphic to a Lie subalgebra of $s o(N)$. Then, it admits a bi-invariant metric, and therefore $\tilde{G}=K \times \mathbb{R}^{k}$, with $K$ compact.

We have that the orbits of $K$ in $M$ are compact submanifolds of $\mathbb{R}^{N}$. So, any of these orbits has a well determined barycenter in $\mathbb{R}^{N}$. Let $B$ be the affine subspace of $\mathbb{R}^{N}$ generated by all of these barycenters. Then, since $K$ is a normal subgroup of $G$, we have that the group $\mathbb{R}^{k}$ acts on $B$, which is left pointwise fixed by $K$. Let $\mathbb{V}$ be the orthogonal complement to $B$, with respect to some point. It is easy to see that the representation of $\rho$ in $I\left(\mathbb{R}^{N}\right)$ can be written in the form: $\rho(k, w)(v, b)=\left(\rho_{1}(k, w)(v), \rho_{2}(w)(b)\right)$, where $(k, w) \in K \times \mathbb{R}^{k}=\tilde{G},(v, b) \in \mathbb{V} \times B=\mathbb{R}^{N}$. Moreover, $\rho_{1}$ is a representation of $\tilde{G}$ into $S O(\mathbb{V})$. (In the above characterization we may replace $B$ by the bigger subset which consists of all points of $\mathbb{R}^{N}$ fixed by $K$.) Therefore, we have proved the following theorem.

Theorem. Let $M=G \cdot v$ be a (noncompact) homogeneous irreducible submanifold of $\mathbb{R}^{N}$, where $G$ is a Lie subgroup of the isometry group $I\left(\mathbb{R}^{N}\right)$ of $\mathbb{R}^{N}$. Then, the universal cover $\tilde{G}$ of $G$ splits as $K \times \mathbb{R}^{k}$, where $K$ is a compact simply connected Lie group. Moreover, the induced representation of $K \times \mathbb{R}^{k}$ into $I\left(\mathbb{R}^{N}\right)$ is equivalent to $\rho_{1} \oplus \rho_{2}$, where $\rho_{1}$ is a representation of $K \times \mathbb{R}^{k}$ into $S O\left(\mathbb{R}^{d}\right)$ and $\rho_{2}$ is a representation of $\mathbb{R}^{k}$ into $I\left(\mathbb{R}^{e}\right)(N=d+e)$.

\section{REFERENCES}

[D] J. Dadok, Polar coordinates induced by actions of compact Lie groups, Trans. Amer. Math. Soc. 288 (1985), 125-137.

[E] J. Erbacher, Reduction of the codimension of an isometric immersion, J. Differential Geometry 5 (1971), 333-340.

[F] D.Ferus, Symmetric submanifolds of Euclidean space, Math. Ann. 247 (1980), 81-93.

[HOT] E. Heintze, C. Olmos and G. Thorbergsson, Submanifolds with constant principal curvatures and normal holonomy groups, Internat. J. Math. 2 (1991), 167-175.

[M] J. D. Moore, Isometric immersions of Riemannian products, J. Differential Geometry 5 (1971), 159-168.

[O1] C. Olmos, The normal holonomy group, Proc. Amer. Math. Soc. 110 (1990), 813-818.

[O2] _ Isoparametric submanifolds and their homogeneous structures, J. Differential Geometry 38 (1993), 225-234.

[O3] _ Homogeneous submanifolds of higher rank and parallel mean curvature, J. Differential Geometry 39 (1994), 605-627.

[PT] R. Palais and C.-L. Terng, Critical point theory and submanifold geometry, Lecture Notes in Math., vol. 1353, Springer-Verlag, Berlin, 1988. 
[S] W. Strübing, Isoparametric submanifolds, Geom. Dedicata 20 (1986), 367-387.

[Te] C.-L. Terng, Isoparametric submanifolds and their Coxeter groups, J. Differential Geometry 21 (1985), 79-107.

[Th] G. Thorbergsson, Isoparametric foliations and their buildings, Ann. of Math. 133 (1991), 429-446.

[V] J. Vargas, A symmetric space of noncompact type has no equivariant isometric immersions into the Euclidean space, Proc. Amer. Math. Soc. 81 (1981), 149-150.

[W] A. West, private communication.

Fa.M.A.F., Universidad Nacional de Córdoba, Ciudad Universitaria, 5000 Córdoba, ARgentina

E-mail address: olmos@mate.uncor.edu 